(Aus der Universitäts-Kinderklinils in Wien [Vorstand: Prof. Dr. C. Pirquot].)

\title{
Zur Symptomatologie und Pathogenese des Oedema scorbuticum invisibile.
}

\author{
Von \\ Arvid Wallgren, \\ Dozent an der Universität Upsala, Schweden. \\ Mit 6 Textabbildungen.
}

(Eingegangen am 1. September 1921.)

Seit langem war man der Ansicht, der Säuglingsskorbut sei durch Skelettveränderungen, und durch Erscheinungen, wie sie bei hämorrhagischer Diathese vorkommen, völlig charakterisiert; über andere Phänomene bekam man in den Handbüchern im allgemeinen keine oder sehr geringe Auskunft. Die vermehrte Anzahl der Skorbutfälle sowohl bei Erwachsenen als auch bei Säuglingen, die in den letzten Jahren vorgekommen sind, haben aber zu fortgesetzten klinischen Studien geführt und mehrere wertvolle Nachträge zur Symptomatologie der Krankheit gegeben. Ich will hier nur den von Fränkel beschriebenen typischen Röntgenbefund der Skelettveränderungen, die bessere Kenntnis der Blutveränderungen, die Capillarwandschwäche und die Herzdilatation bei dem Säuglingskorbut erwähnen. Ähnliche Beobachtungen hatte man zwar schon früher gemacht, aber die in der älteren Literatur vorkommenden diesbezüglichen Angaben waren vergessen oder überhaupt nicht beachtet worden. In der älteren Literatur war auch ein anderes Krankheitsphänomen erwähnt, welchem man in der letzten Zeit ein gewisses Interesse gewidmet hat, nämlich eine Störung des Wasserhaushaltes.

Während der Kriegsjahre hatte man oftmals Wasseranschwellungen der skorbutkranken Kriegsgefangenen beobachtet; Wasserschwellungen, die bisweilen so hochgradig gewesen sind, daß man sie als eine den Skorbut komplizierende Ödemkrankheit auffaßte. Diese Tendenz zur Wasserretention ist aber nicht eine Eigentümlichkeit, die nur den Skorbut der Erwachsenen betrifft. Wenn man nach Zeichen einer ähnlichen Störung bei skorbutkranken Säuglingen sucht, so kann man sie auffallend of $t$ nachweisen. L. Meyer sieht auch in dem Morbus Barlow eine der Krankheiten, die mit einer Tendenz zur Wasserretention einhergeht und in dieser Hinsicht z. B. der exsudativen Diathese ähnelt. 
Hess ist vielleicht als erster auf diese Frage näher eingegangen. Er schreibt hierüber: „In our experience subcutaneous edema is not only one of the most constant phenomena in the symptomatology and pathology of this disorder (infantil scurvy), but deserves special consideration because it appears in a peculiar clinical form and frequently is one of the earliest indications of threatening scurvy. "Daß diese Wasserretention von den älteren Autoren übersehen worden ist oder jedenfalls für sie eine so geringe Rolle in der klinischen Symptomatologie dieser Krankheit gespielt hat, ist nach Hess darauf zurückzuführen, daß die Wasserretention nicht wie ein gewöhnliches Ödem aussieht; es hinterläßt nämlich keine Fingerabdrücke. In seiner Arbeit über die Barlowfrage kommt Nobel auch zu der Überzeugung, daß bei dieser Krankheit eine Wasserretention besteht, die sich nicht als gewöhnliches Ödem kundgibt, sondern die sich nur durch große Körpergewichtsschwankungen entlarvt. Um diesen für die gewöhnliche klinische Untersuchung also nicht nachweisbaren pathologischen Wassergehalt des Körpers. zu charakterisieren, schlägt Nobel vor, sie als Oedema scorbuticum invisibile zu bezeichnen.

Durch Professor Pirquet angeregt, diese Frage näher an dem Barlowmaterial der Wiener Universitäts-Kinderklinik, das bis jetzt etwas mehr als 20 Fälle umfaßt, zu untersuchen, will ich hier über meine Beobachtungen berichten und daran einige Worte über die Pathogenese dieses Symptoms anschließen. Die Studien, die ich über diese Frage gemacht habe, sind hauptsächlich auf Untersuchungen am Krankenbett gegründet.

Das vielleicht am meisten ins Auge fallende Symptom, das schon von Nobel erwähnt wurde, sind unmotivierte große Gewichtsschwankungen. Man ist ja der Ansicht, daß jeder plötzliche oder unaufgeklärte Gewichtsanstieg oder Gewichtssturz auf eine Änderung des Wasserbestandes zurückzuführen sei, was ja a priori in Anbetracht des großen Wassergehaltes des Säuglingsorganismus sehr wahrscheinlich ist. Große Gewichtsschwankungen sind jedoch Erscheinungen, die bei vielen verschiedenen Erkrankungen vorkommen und die auf verschiedenen ätiologischen Faktoren beruhen können. Eine besondere Durchforschung jedes einzelnen Falles in dieser Hinsicht ist also in erster Linie indiziert. Aber auch mit größter Kritik beurteilt, findet man oftmals Gewichtsschwankungen bei dem Säuglingsskorbut, die sich nicht durch irgendwelche andere Störungen völlig erklären lassen und daher als Schwankungen des Wassergehaltes gedeutet werden müssen.

Diese Gewichtsschwankungen sind also nicht als ein eben für diese Krankheit typisches Phänomen zu betrachten. Die Gewichtskurve zeigt im allgemeinen auch nicht einen für Säuglingsskorbut eigenartigen 
Typus. Diese Gewichtsstürze sind am gewöhnlichsten während des floriden Stadiums der Krankheit vorhanden. Dieses Verhältnis hängt wohl damit zusammen, daß die hämorrhagische Diathese mit den Skelettveränderungen einerseits und der Störung des Wasserhaus. haltes andererseits gewissermaßen parallel verlaufen. (siehe unten). Große Gewichtsstürze können aber auch vorhanden sein zu einer Zeit, wo die klinischen Skorbutsymptome schon vorüber sind. Als Beispiele will ich hier zunächst nur zwei Fälle mitteilen.

Fall $1^{1}$ \}. Kari D., 9 Monate alt. Aufgenommen am 2. III. 1921. Entlassen am 15. III. 1921.

A uszug a us der Anamnese: Erste rechtzeitige Zangengeburt. 6 Wochen Brust, dann Allaitement mixte mit 3stïndlichen Mahlzeiten bis Ende des 2. Lebensmonats. Seit September 1920 täglich 1 Liter Milch, der teils zu Milchverdünnungen mit Zucker, teils zu Mehlpapperl verwendet wurde. Hier und da Spinat und Erdäpfel. Acht Zähne. Sitzt noch nicht. Erbrechen nach jeder Mahlzeit seit 1 Monat. Jetzt 1 Tag lang Diarrhöe. Seit 14 Tagen Sohmorzen in der linken Hand und in beiden Beinen. Thorax empfindlich.

Auszug aus dem Status: Normaler Turgor und Fettgehalt. Vereinzelte Petechien an der Brust, Bauch und linkem Oberarm. Haut über beiden Kniegelenken gespannt. und glänzend. Hält die Beine ruhig. Unbedeutende Druckempfindlichkeit der Beine. Zahnfleisch des

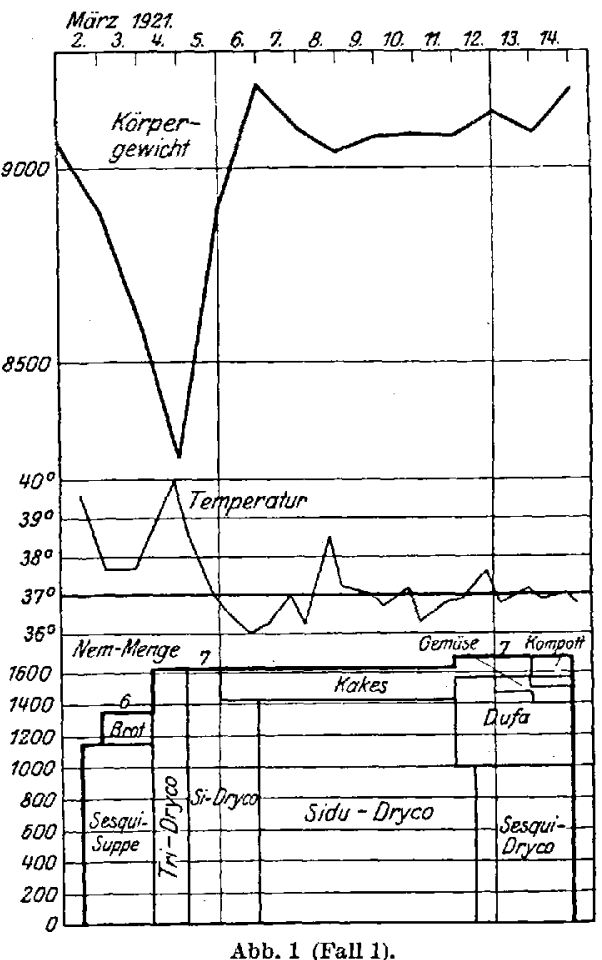

Abb. 1 (Fall 1). Oberkiefers stark aufgelockert, geschwollen, düsterrot verfärbt, leicht blutend. Geringgradige Rachitis. Doppelseitige Otitis media. Stuhl etwas blutig-schleimig.

Decurs us morbi: Am 5. III. Fieber bis $40^{\circ}$. Trommelfell gerötet, Erbrechen. Gewichtssturz von $800 \mathrm{~g}$ in 3 Tagen. Zusatz von Zitronensaft zu der Nahrung, Nahrungsverminderung. Dann Gewichtsanstieg von $900 \mathrm{~g}$ in 2 Tagen. Die Barlowerscheinungen sind während dieser Zeit schnell zurückgegangen. Schon am 5. III. wurde bemerkt, daß das Kind seine Beine viel besser bewegte; das Zahnfleisch besserte sich ebenfalls, und am 7. III. batte das Kind keine Sehmerzen mehr. Der oben erwähnte gewaltige Gewichtssturz, in 1 Tag schon repariert, ist vielleicht

1) Die Krankengeschichten der Fälle 1,3 und 6 sind ausführlicher in der Arbeit von Nobel und Wagner ,Trockenmilch in der Kinderernährung", die der Fälle 2, 4 und 5 in Nobels Arbeit über die Barlowfrage schon erschienen. Die Abbildungen 2 (9), 4 (1) und 5 (10) entstammen der letztgenannten Arbeit. 
durch die Otitis media ausgelöst; die Magendarmerscheinungen waren nicht so ausgesprochen, daß sie allein den Gewichtssturz erklären konnten.

Fall 2. Karl E., 20 Monate alt. Aufgenommen am 30. X. 1920. Entlassen am 1. IV. 1921.

A uszug a us der Anamnese: Zweite, rechtzeitige normale Geburt. 12 Monate Mutterbrust. Ab 6. Monat Papperl aus Milch, Zucker und Reis oder Grieb. Bis zum 12. Monat hatte die Mutter zur Bereitung des Papperls frische Milch, dann nur Kondensmilch, dann wieder täglich $1 / 4$ Liter frische Milch genommen. Vom 12. Monate an gemischte Kost. Zähne mit 10 Monaten, Laufen und Sprechen mit 15 Monaten. Vor 5 Monaten fiel der Mutter zum ersten Male auf, daß das sonst sehr lebhafte Kind sich nur mehr dadurch fortbewegen konnte, daB es sich

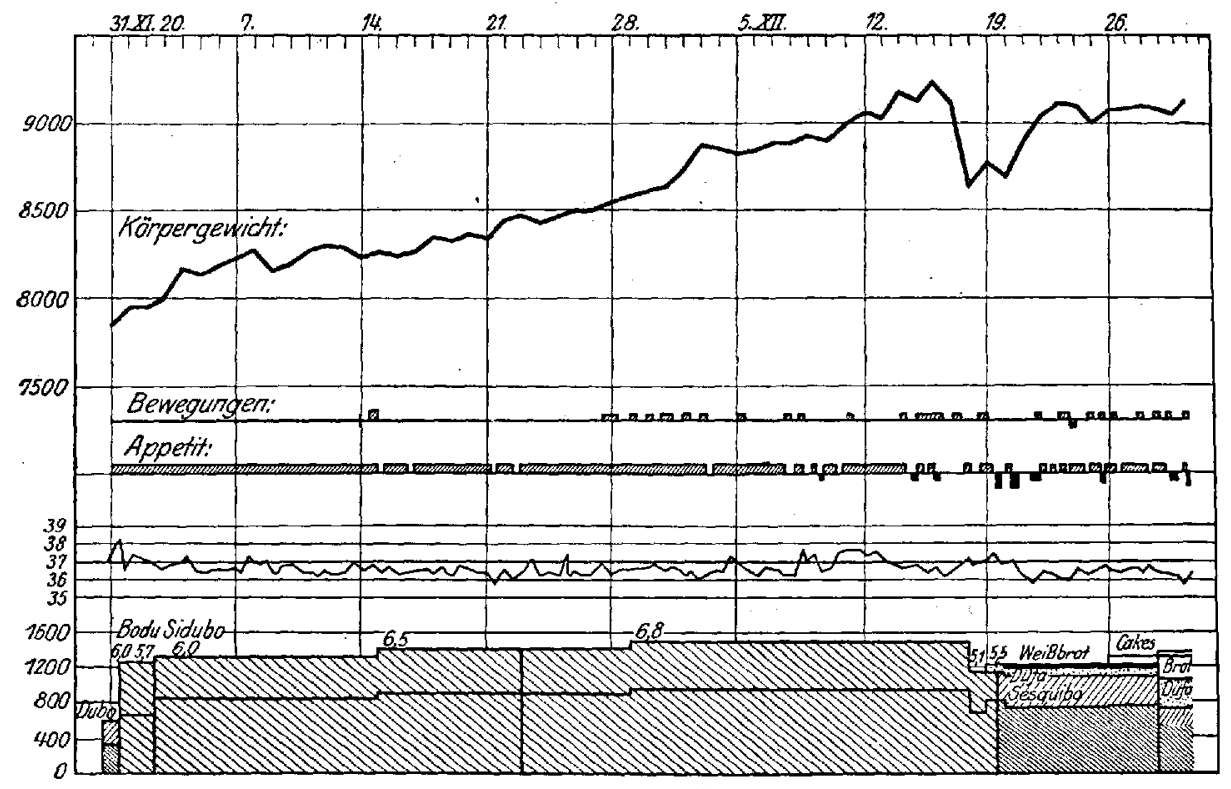

Abb. 2 (Fall 2).

anhielt. Allmählich zunehmende Appetitlosigkeit, das Kind hörte auf zu gehen. Seit einigen Wochen hat das Kind Schmerzen in den Armen und Beinen.

Auszug aus dem Status: Fettgehalt und Turgor normal. Punktförmige Hämorrhagien an beiden Oberarmen, Unterarmen, rechtem Oberschenkel und am Rücken. Rachitischer Rosenkranz, Knöchel am Handgelenk aufgetrieben. Das rochte Bein wird im Hüft- und Kniegelenk gebeugt gehalten, jede aktive Bewegung wird ängstlich vermieden. Bei passiven Bewegungen greift das Kind ängstlich mit beiden Händen auf den Unterschenkel und weint. Das rechte Kniegelenk deutlich geschwollen, Haut darüber gespannt, deutliches ödem. Schwellung des Zahnfleisches, entsprechend den Zähnen am Oberkiefer, dasselbe livid gerötet, leicht blutend. Zahnfleischveränderungen am Unterkiefer geringer. Harnbefund negativ. Blutbefund: Rote Blutkörperehen 4,600,000, weiße 18,400, Sahli 77\%, Blutungszeit 4 Minuten, Gerinnungszeit normal. Herzverbreiterung besonders nach links bis über die Mammillarlinie.

Decursus morbi: Das Kind bekam rom Anfang des Spitalaufenthaltes an Milch, die eine Stunde lang vom Zeitpunkt des Kochens an gerechnet gekocht 
wurde, zunächst in doppelter Konzentration, dann in $1 / 1 / 2$ facher Konzentration. Die Barlow-Erscheinungen besserten sich allmählich; nach einer Woche waren die Zahnfleischveränderungen im Rückgang, die Schmerzen im linken Bein etwas geringer. Nach etwa 14 Tagen waren die Schmerzen völlig geschwunden, und das Zahnfleisch war völlig normal. Ab 12. XI. bekam das Kind gemischte Kost. Das Körpergewicht hatte während des Rückgangs der klinischen Barlowerscheinungen einen sehr unbedeutenden Anstieg gezeigt. Nach einem Monat stieg es rascher, jedoch trat am 18. XI. ein Gewichtssturz von $530 \mathrm{~g}$ ein, der nicht durch eine schwere Ernährungsstörung hervorgerufen wurde. Dann wieder Ansteigen des Gewichtes bis zur Entlassung.

In einem auffallend großen Teil der Fälle haben sich die Gewichtskurven in einer eigenartigen Weise verhalten. Man findet nämlich in einer Zeit, wo die klinischen Skorbutsymptome abgeklungen sind, also während des Reparationsstadiums der Krankheit, eine leichte Senkung der Gewichtskurve. Für diese findet sich in den von mir untersuchten Fällen gar keine genügende Erklärung. Es liegt keine Magen-Darmstörung vor, die diesen Gewichtsabfall genügend erklären könnte, es war keine andere interkurrente Krankheit vorhanden, auch keine Diätänderung im Sinne einer nährwertärmeren Kost. Dieser Gewichtsabfall dauert einige Tage bis zu einer Woche, dann tritt noch mals eine Steigerung des Gewichtes ein. Hier einige Beispiele.

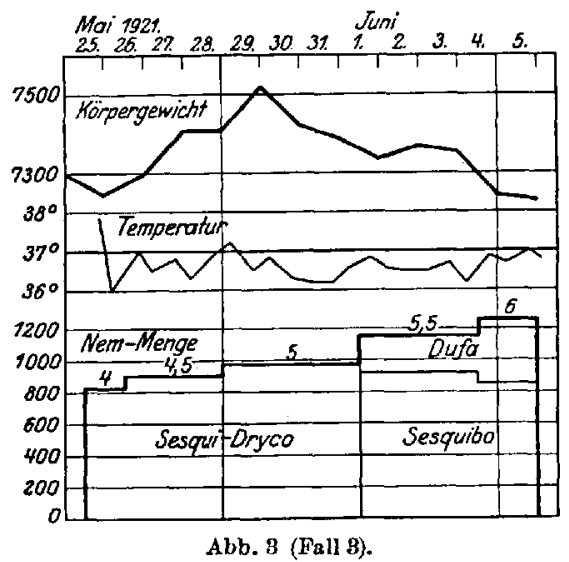

Fall 3. Emilie T., 8 Monate alt. Aufgenommen am 25. V. 1821. Entlassen am 6. VI. 1921 .

A uszug aus der Anam nese: Zweite rechtzeitige Geburt. Bis zum 3. Monate an der Brust, später Flasche, 2 Monate mit verdünnter Kuhmilch, dann mit Kondensmileh und Nestle. Seit etwa 2 Monaten Spinat und Rindssuppe. Ein Zahn. Seit etwa 14 Tagen bemerkt die Mutter, daß das Kind, das schon allein stehen konnte, beim Stehen zusammenfiel und schreit. Kein Appetit.

Auszug aus dem Status: Blutgehalt und Turgor normal. Fettgehalt reichlich. Zahlreiche Petechien an der Außenseite des linken Unterschenkels und der rechten Oberbauchgegend. Gesicht und Extremitäten leichtödematös. Geringgradige Rachitis. Die Beine werden ruhig gehalten; leichte Druckempfindlichkeit der Kniee. Keine Zahnfleischveränderungen. Im Röntgenbild typischer Barlow.

Decurs us morbi: Das Kind bekam zuerst ein Trockenmilchpräparat (Dryco) als $1 \%$ fache Nahrung. Die recht geringgradigen klinischen Erscheinungen gingen dabei schnell zurück, und am 30. V. war das Kind schon symptomenfrei Bis jetzt ein normaler Gewichtsanstieg von $7300 \mathrm{~g}$ bis zu $7520 \mathrm{~g}$ Der Turgor wurde jetzt als übernormal bezeichnet. Dann trat ein Abfall des Gewichts ein, der bis zur 
Entlassung dauerte und nicht durch eine Ernährungsstörung gedeutet werden kounte.

Fall 4. Otto M., 8 Monate alt. Aufnahme 18. VI. 1920, Spitalsaufenthalt bis 16. VII. 1920.

Auszug a us der Anamnese: Dritte rechtzeitige normale Geburt. Wegen Wolfsrachen seit der Geburt künstliche Ernährung. Anfangs etwas gezuckerte

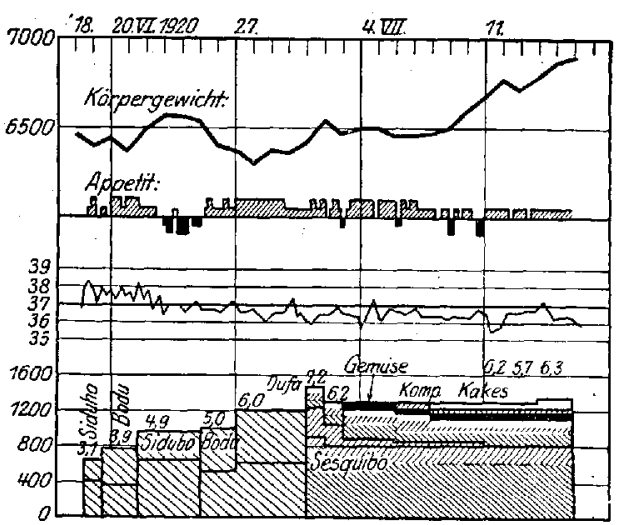

Abb. 4 (Fall 4). Halbmilch, mit 2 Monaten wurde Nestlemehl in die Milch eingekocht. Mit 3 Monaten einmal täglich, alsbald zweimal täglich Papperl aus Grieß 3 und Mebl. 14 Tage vor der Aufnahme nur gezuckerte Halb. milch. Zwei Zähne. Nach dem 6. Monate wurden bei dem Kinde Unruhe und Sohmerzen bei Bewegungen beobachtet. Alsbald konnten Schmerzen in das angeschwollene linke Kniegelenk lokalisiert werden. Mit ca. 7 Monaten Schwellung des rechten Kniegelenkes. Seit 14 Tagen vor der Aufnahme zunehmende Unruhe, Schreien, Apathie, Appetitlosigkeit, Zunahme der Kniegelenksschwellung.

A uszug a us dem Status: Blaß. Fettgehalt und Turgor normal. Deutliche Rachitiszeichen. Beide Kniegelenke geschwollen, besonders das linke, ebenso beide Fußrücken, wie die beiden unteren Extremitäten im ganzen. Beide Beine bei Bewegung schmerzhaft, besonders das linke; leichte, bläulich-zyanotische Verfärbung

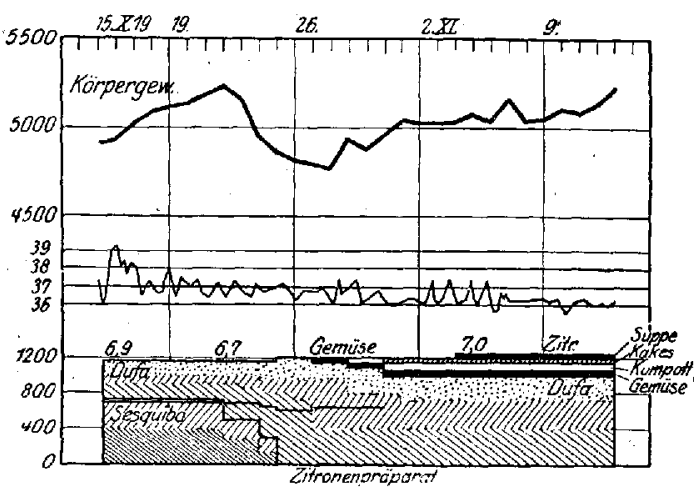

Abb. 5 (Fall 5)entsprechend den zwei unteren Schneidezähnen. Zahnfleisch aufgelockert.

Decurs us morbi: Ernährung durch die ersten 13 Tage ausschließlich mit durch Erhitzen konzentrierte Milch, ansteigend von $3-6$ dnsq. Dann gemischte Kost. Schon am 21. VI. war die Gingiva gebes. sert, Fußrücken weniger geschwollen, Schmerzhaftigkeit des linken Beines geringer. Am 24. VI. hatte das Kind keine Schmerzen mehr. Am 25. VI. wurde notiert, daß die Schwellung und der Glanz über den Fußrücken und Unterschenkel weit geringer waren und daß vermehrter Harnabgang beobachtet wurde. Jetzt trat ein Gewichtsabfall ein, im ganzen $280 \mathrm{~g}$ in 5 Tagen. Dann wieder allmähliches Ansteigen der Gewichtskurve bis zur Entlassung.

Fall 5. Kurt B., 8 Monate alt. Aufgenommen am 15. X. 1919. Entlassung am 13. XI. 1919. 
A uszug a us der Anamnese: Erste, rechtzeitige normale Geburt. Bekam verdünnte Milch und Mehlpapperl. In bereits abgekochter Milch wurde Zwieback eingekocht. Ein Zahn. Seit 3 Monaten Schmerzen bei Streckung der Beine, seit 4 Wochen Schwellung des linken Beines.

Auszug aus dem Status: Blaß, geringer Fettgehalt und Turgor. Zah: reiche, punktförmige Hämorrhagien an der Kopfhaut und auf dem Stamm. Thorax vorne eingesunken. Hält den linken Unterschenkel unbeweglich. Schwellung in der Kniegegend, Haut darïber glänzend. Keine Zahnfleischveränderungen. Blutbefund: Rote Blutkörperchen 3,800,000, weiße 3800, Sahli 45. Röntgenbefund: Morbus Barlow; subperiostales Hämatom am unteren Teil des linken Femur.

Decursus morbi: Behandlung: gemischte Kost, Dufa, Sesquibo' ${ }^{1}$ ), Gemüse, Zitronenpräparat. Unter allmählichem Gewichtsanstieg gingen die Erscheinungen schnell zurick, die Hautblutungen waren im Rückgang, die Schmerzen geringer. Dann trat vom 22. X. ab ein Gewichtsabfall ein, der bis zum $27 . \mathrm{X}$. dauerte und im ganzen $460 \mathrm{~g}$ betrug. Gleichzeitig besserten sich die Skorbutsymptome noch mehr, und die Schwellung der Beine nahm ab von $16,5 \mathrm{~cm}$ bis $15,5 \mathrm{~cm}$. Dann wieder eine allmähliche Zunahme des Gewichts.

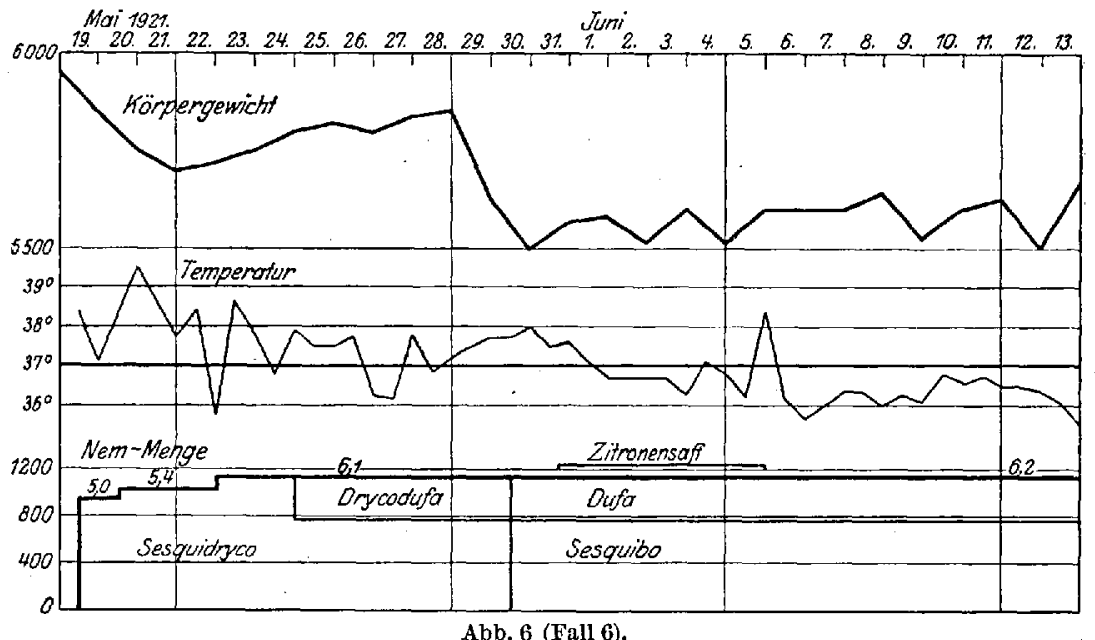

Fall 6. Erik N., 8 Monate alt. Aufnahme 19. V. 1921. Entlassen am 25. VI. 1921.

A uszug aus der Anamnese: Erste, rechtzeitige normale Geburt. Aufgezogen mit durch Reisschleim verdünnter Milch mit Zuckerzusatz. Mit $2 \frac{1}{2}$ Monaten außerdem Nestle. Die Milch wurde eine Viertelstunde gekocht. In den letzten 14 Tagen auch Gemüse und jeden zweiten Tag Eier. Zwei Zähne. Seit 5 Wochen hat das Kind Schmerzen in den Beinen, die unbeweglich gehalten werden.

A uszug a us dem Status: BlaB, Fettgehalt und Turgor normal. Zerstreute Petechien über dem Stamm und der Glutealgegend. Beide Beine in Ruhestellung. Haut über den Kniegelenken gespannt und glänzend. Bei passiven Bewegungen besonders links, Schmerzäußerungen. Deutliche Rachitiszeichen. Zahnfleisch livid verfärbt.

1) Sesquibo = anderthalbfache Nahrung von gezuckerter Kuhmilch, Dubo $=$ eine Doppelnahrung von gezuckerter Kuhmilch, Dufa $=$ eine Doppelnahrung von Grießbrei. 
Decursus morbi: Das Kind bekam zuerst Trockenmilchpräparat (Dryco) in $11 / 2^{-}$resp. 2 facher Konzentration. Die Barlowsymptome gingen in dieser Zeit schnell zurück, und am 28. V. war das Zahnfleisch beinahe normal. Schmerzhaftigkeit der unteren Extremitäten kaum vorhanden. Unter fortwährender Besserung der Skorbuterscheinungen, aber vermindertem Turgor trat am 29. V. ein Gewichtsabfall ein, der zwei Tage dauerte und im ganzen $350 \mathrm{~g}$ betrug. Nachber ein sehr flacher Verlauf der Gewichtskurve. Seit 1. VI. vollkommene Symptomfreiheit. Vom 30. V. ab wegen Mangels an Trockenmilch Übergang zu friseher Milch.

Hess hat auch in einigen Fällen ein etwas ähnliches Verhalten der Gewichtskurve während der Besserung beobachtet. Er spricht von einem unmotivierten Gewichtsstillstand, wenn die antiskorbutische Kost gegeben wird und die Besserung der Krankheit deutlicher wird. In dem oben erwähnten Fall 2 kann man etwas Ähnliches beobachten. In diesem Falle war die Verflachung der Gewichtskurve während der Besserung der klinischen Skorbutsymptome auffallend. Gut begründet ist, scheint mir, die von Hess ausgesprochene Ansicht, daß der fehlende Gewichtsanstieg im Anschlu $B$ an die Besserung der Krankheit auf einen während dieser Zeit eintretenden Wasserverlust des Organismus zurückzuführen ist. Er hat nachweisen können, daß in diesem Stadium der Krankheit eine vermehrte Diurese vorhanden ist, in dem Höhestadium dagegen eine Oligurie. Einige Male ist die Harnmenge auch in den von mir zusammengestellten Fällen gemessen worden, und dabei hat man eine Steigerung derselben im Reparationsstadium konstatiert (siehe Fall 4). Kurz gesagt, es scheint, als ob das Kind im floriden Stadium der Krankheit Wasser aufspeicherte, das es während des Reparationsstadiums wieder ausscheidet. Es kommt dadurch zu einer Harnflut und die Gewichtskurve stcigt nicht. Daß dieser fehlende Anstieg oder Abfall des Gewichtes darauf zurückzuführen ist, dafür spricht auch der Umstand, daß der Allgemeinzustand der Kinder nicht im ungünstigen Sinne beeinflußt wird; im Gegenteil werden sie während dieser Zeit eher besser.

Wie aus den oben referierten Krankengeschichten hervorgehen dürfte, waren in einigen Fällen Ödeme beobachtet worden (Fall 2 und 3). In diesen Fällen ist es ja ohne weiteres klar, daß eine abnorme Wasserretention vorhanden war. So einfach liegt die Sache nur in Ausnahmsfällen. Für gewöhnlich hat man nicht die Wasseraufspeicherung als Ödem nachweisen können. Es ist ja ein großer Unterschied zwischen latentem und manifestem Ôdem, und in vielen Fällen gibt es wahrscheinlich keine Übergänge zwischen diesen beiden Formen der Wasserretention. Es ist ein wesentlicher Unterschied zwischen ihnen; bei latentem Ödem ist die Störung der Wasserhaushaltes auf das intracellulare, assimilierte Wasser beschränkt, bei klinischem Ödem ist die Assimilationsfähigkeit der Zellen herabgesetzt oder ihre Funktionsbreite schon überschritten. Aber auch das latente Ödem beim Säug. 
lingsskorbut ist, wie Hess hervorgehoben hat, bisweilen doch klinisch nachweisbar, und zwar als vermehrter Turgor. Ähnliche Beobachtungen sind auch unter meinen Fällen vorhanden (siehe Fall 3). In der Wiener Universitäts-Kinderklinik wird jede zweite Woche u. a. der Turgor der Säuglinge festgestellt, und zwar nach fünf verschiedenen Graden. Die Verschiedenheit zwischen der normalen und der pathologischen Gewebsspannung ist ja so groß, daß man keine besonderen Apparate braucht, um sie festzustellen. Mittels Palpation kann man sich leicht von dem Grad der Spannung überzeugen. So wird auch die Schätzung des Turgors in der Säuglingsstation durch Palpation gemacht, und zwar immer vom Stationsarzt. Bisweilen kann man so feststellen, daB der Turgor bei den Barlowfällen zu Beginn des Spitalsaufenthaltes sich vermindert (siehe Fall 6), in der Zeit zwisehen Höhepunkt und Besserung der Krankheit, in einer Zeit also, wo wir die Gewichtssenkung gefunden haben. Diese Turgorverminderung will ich mit Hess als Ausdruck einer während des Reparationsstadiums der Krankheit eintretende Ausscheidung pathologisch aufgespeicherten Wassers deuten.

Davon, daß eine Abschwellung des Körpers während des Reparationsstadiums zustande kommt, kann man sich auch direkt durch das Meßband überzeugen. Eine Verminderung z. B. des Beinumfanges um 1-2 cm ist keine Seltenheit (siehe z. B. Fall 5). Diese Verminderung des Beinumfanges darf man aber nicht ohne weiteres auf eine geschwundene Wasserschwellung zurückführen. Es ist ja verständlich, daß es sehr schwierig sein kann, intra vitam zu beurteilen, ob eine Schwellung der Extremitäten bei Säuglingsskorbut auf Ödem oder auf periostalem Hämatom beruht. Im allgemeinen scheint man geneigt $\mathrm{zu}$ sein, ohne weiteres jede Extremitätenanschwellung bei Barlow als eine Blutung zu deuten. Meiner Ansicht nach geht man aber dabei zu weit. Die Schwellung betrifft oft die Weichteile außerhalb des Gebietes der subperiostalen Blutung, oft kommt sie auch am Fußrücken vor usw.. Die wahre Ursache dieser Anschwellung kann man wohl nur durch die Obduktion nachweisen und, wenn man die in der Literatur publizierten Sektionsberichte durchliest, so findet man dort Angaben von ödematöser Durchtränkung der Muskulatur, mit oder ohne gleichzeitige subperiostale Blutungen (Bardt und Edelstein, Epstein, Stoeltzer, Fürst, Asch off und Koch, Barlow). Da die Wasserdurchtränkung der Muskulatur vorhanden sein kann ohne gleichzeitige Blutung, so kann man es nicht ohne weiteres als Kollateralödem auffassen. Gerade diese Lokalisation des Gewebswassers erklärt nach Hess, daß man sie so lange nicht beachtet und daß man die Störung des Wasserhaushaltes bei diesen Kindern überhaupt übersehen hat.

Durch alle hier erwähnten Beobachtungen, Ödeme während des Höhestadiums der Krankheit, reparatorische Gewichtssenkung mit 
Verminderung des Turgors, Harnflut und Abschwellung der Glieder, dürfte es als festgestellt gelten, daß bei Barlow eine Tendenz zur Aufspeicherung von Wasser im Organismus vorhanden ist. Warum es so ist, und ob diese Wasserretention zum klinischen Bilde des Säuglingsskorbuts als solchem gehört, oder ob sie nur als gewöhnliches alimentäres Ödem aufzufassen ist, kann nur Gegenstand von Vermutungen sein.

Wenn es sich um Erwachsene handeln würde, dann hätte man in erster Linie an ein Nierenleiden oder an Herzschwäche zu denken. Da es sich aber um Säuglinge handelt, müssen wir andere Erwägungen bezüglich der Ätiologie der Wasserretention in Betracht ziehen. Die Erscheinung von Ödemen bei Säuglingen ist ja nichts Ungewöhnliches. Es ist bekannt, daß diese, im Gegensatz zu den Ödemen der Erwachsenen, nur ausnahmsweise von einer renalen oder kardialen Ätiologie sind. Ein großer Teil ist alimentär bedingt, $d$. h. sie können hervorgerufen werden durch ungeeignete Nahrung, und sie können durch gut zusammen. gesetzte Nahrung zum Versehwinden gebracht werden.

Im Fall 2 und auch in noch einigen, hier nicht referierten Fällen war eine Herzdilatation vorhanden, ein Symptom beim Sänglings. skorbut, von dem verschiedone Autoren berichtet haben. Es ist jedoch höchst unwahrscheinlich, daß die Wasserretention auf einer Herzschwäche, deren klinisches Herzsymptom eine Dilatation ist, beruht, denn wir müssen als eine Eigentümlichkeit. der Wasserretention bei dieser Krankheit feststellen, daß sie nicht als kardiale Ödeme imponieren. Viel mehr erinnert ihre Lokalisation an Nephritis. Es liegt nun nahe, die Wasserretention daher auch als nephritisch aufzufassen, besonders weil es ja eine gewöhnliche Erscheinung ist, daß Erythrocyten, gegebenenfalls auch andere Formelemente, im Harn auftreten. Bisweilen kommt es auch vor, daß die Nierenblutung beinahe das einzige klinische Symptom des Skorbuts ist; daß dann die Differentialdiagnose gegenüber Nephritis bei gleichzeitigem Ödem oft Schwierig. keiten bereiten kann, ist sclbstverständlich. Es dürfte auch unmöglich sein, klinisch einen Untersehied zwischen hämorrhagischer Nephritis und einer skorbutischen Nierenblutung zu machen. Die Schwierig. keiten können noch größcr werden dadurch, daß die Wasserretention bei Säuglingsskorbut eines der frühesten Symptome ist (Hess). Die Erfahrung lehrt aber, daß die Nierenveränderungen, die man beim Säuglingsskorbut nachweisen kann, nicht nephritischer Art sind. Beinahe ausnahmslos sind die Nieren bei der Sektion frei, höchstens hat man kleine parenchymatöse Blutungsherde gefunden (Fränkel), die nicht die Ursache der Ödeme sein können.

Hess ist jedoch geneigt, die Oligurie und die Wasserretention während der Krankheit auf ein mangelhaftes Ausscheidungsvermögen 
der Nieren zurückzuführen, eine Funktionsschwäche, die er durch die diuretische Wirkung des Zitronensaftes aufheben konnte. Es ist aber eine Zufälligkeit, wenn Hess gefunden hat, daß gerade der Zitronensaft das aufgespeicherte Wasser hinaustreibt, denn dieselbe Wirkung wurde in der Wiener Kinderklinik durch verschiedene antiskorbutische Nahrungen hervorgerufen, nach der darauf folgenden Gewichtssenkung zu urteilen. Ich glaube auch nicht, daß Hess das Richtige getroffen hat, wenn er meint, daß das antiskorbutische Vitamin an sich ein wahres Diuretikum ist. Man hat eine viel näher liegende Erklärung der Diurese während der Besserung der Krankheit; da der Skorbut gebessert wird, ungeachtet durch welche Behandlung, so kommt es ja zu einer Besserung jedes einzelnen Symptoms, und zwar auch in der Störung des Wasserhaushaltes, die also geringgradiger wird. Das aufgespeicherte Wasser wird dabei wieder ausgeschieden, daher dio scheinbar diuretische Wirkung der Antiskorbutica. Meiner Ansicht nach liegt in dieser Tatsache gar kein Grund, an eine Funktionsstörung der Nieren zu denken, ebensowenig wie die Nieren die Ursache der Wasserretention bei der Ödemkrankheit sind.

Bei Säuglingen ist es ja eine altbekannte Tatsache, daß $B$ eine während längerer Zeit verabreichte einseitige Kohlenhydraternährung, besonders Mehlnahrung, bedeutende Wasseransammlungen im Körper veranlassen kann. Wir wissen, daß es nahe Beziehungen zwischen dem Kohlenhydratgehalt der Nahrung und dem Wasseransatz im Körper gibt. Der Anstieg oder der Sturz der Gewichtskurve," der Nachweis palpabler Oddeme während einer einseitigen Kohlenhydraternährung läßt uns vermuten, daß es bei dieser Nahrung zu einer Wasserretention kommt, die für das Wohlbefinden des Kindes nicht günstig ist. Auch experimentell hat man nachweisen können (Freund), daß die Kohlen. hydrate ebenso wie das $\mathrm{NaCl}$ eine Neigung zur Wasserretention erzeugen. Nun ist es ja bekannt, daß viele Autoren seit langem hervorgehoben haben, daß sich eine einseitige oder zu reichliche Kohlenhydraternährung so häufig in der Anamnese skorbutkranker Säuglinge findet, daß man sie als einen disponierenden Faktor für die Entstehung dieser Krankheit aufgefaßt hat. Es liegt daher nahe, die skorbutische Wasserretention als einen Mehlnährschaden aufzufassen. In seiner oben erwähnten Arbeit hebt auch Nobel die reichliche Kohlenhydratdiät der Barlowkinder hervor, eine Tatsache, die ich auch in anderon Fällen konstatieren konnte. Aber so liegen die Verhältnisse doch nicht immer, und die Kohlenhydraternährung ist übrigens in keinem Falle so einseitig gewesen, wie man bei MehInährschaden im allgemeinen findet. Es scheint mir daher nicht wahrscheinlich, daß ein Übermaß an Kohlenhydraten in der Nahrung einzig und allein für die Wasserretention beim Säuglingsskorbut verantwortlich sei. Daß man daher auch mit 
einer erhöhten Tendenz zur Wasseraufspeicherung überhaupt bei dieser Krankheit zu rechnen hat, dürfte sehr wahrscheinlich sein.

Kann aber also eine einseitige Kohlenhydraternährung allein in meinen Fällen die Wasserretention nicht erklären, so ist vielleicht die Ursache anstatt dessen in einem allzu großen Salzreichtum der Nahrung oder in einer Störung des Salzstoffwechsels zu suchen. Es ist ja wohl bekannt, daß auch salzreiche Kost bei Säuglingen Wasserretention bewirken kann (Freu nd). Um dieser Frage näher zu treten, waren gewisse Stoffwechseluntersuchungen von großem Wert. Leider habe ich keine Gelegenheit gehabt, eine diesbezügliche Untersuchung bei diesen Kindern zu machen. In der Literatur sind bis jetzt nur zwei Arbeiten über den Mineralstoffwechsel beim Säuglingsskorbut publiziert worden, die eine von Lust und Kloc man, die andere von Frank. Nur die erstgenannte von diesen Arbeiten betrifft auch den NaCl-Umsatz.

\begin{tabular}{|c|c|c|c|c|c|c|c|}
\hline \multirow{2}{*}{ Verfasser } & \multicolumn{4}{|c|}{$\begin{array}{l}\text { I. Während des Höhestadiums } \\
\text { der Krankheit }\end{array}$} & \multicolumn{3}{|c|}{$\begin{array}{l}\text { II. Während des Reparations- } \\
\text { stadiums }\end{array}$} \\
\hline & & Einnahme & $\mid \begin{array}{c}\text { Aus- } \\
\text { scheidung }\end{array}$ & Retention & Einnahme & $\begin{array}{c}\text { Aus- } \\
\text { scheidung }\end{array}$ & Retention \\
\hline & $\mathrm{aO} \ldots$ & & & & & 8,677 & $-2,2099$ \\
\hline & $\begin{array}{l}\mathrm{P}_{2} \mathrm{O}_{5} \ldots . \\
\mathrm{Cl} \ldots \\
\text { Gesamtasche }\end{array}$ & $\begin{array}{r}8,038 \\
4,228 \\
26,611\end{array}$ & $\begin{array}{r}5,582 \\
3,936 \\
20,250\end{array}$ & $\begin{array}{l}+2,456 \\
+0,292 \\
+6,361\end{array}$ & $\begin{array}{r}8,501 \\
2,983 \\
30,863\end{array}$ & $\begin{array}{c}9,901 \\
5,0 \\
48,3\end{array}$ & $\begin{array}{r}1,40 \\
-2,017 \\
-17,437\end{array}$ \\
\hline Frank I & $\begin{array}{ll}\mathrm{CaO} & \cdots \\
\mathrm{P}_{2} \mathrm{O}_{5} & \cdots\end{array}$ & $\begin{array}{l}1,9761 \\
4,5315\end{array}$ & $\begin{array}{l}1,5386 \\
3,4595\end{array}$ & $\begin{array}{l}+0,4375 \\
+1,0720\end{array}$ & $\begin{array}{l}2,2215 \\
4,8858\end{array}$ & $\begin{array}{l}2,0411 \\
3,4816\end{array}$ & $\begin{array}{ll}+ & 0,1804 \\
+ & 1,4042\end{array}$ \\
\hline Frank II & $\left\{\begin{array}{l}\mathrm{CaO} \cdots \cdots \\
\mathrm{P}_{2} \mathrm{O}_{5} \ldots \ldots \\
\text { Gesamtasche }\end{array}\right.$ & $\begin{array}{c}1,9545 \\
4,2476 \\
12,1375\end{array}$ & $\begin{array}{l}0,8577 \\
1,7879 \\
8,6123\end{array}$ & $\begin{array}{l}+1,0968 \\
+2,4597 \\
+3,5252\end{array}$ & $\begin{array}{r}2,0145 \\
3,7298 \\
12,3870\end{array}$ & $\begin{array}{r}3,0926 \\
2,5658 \\
12,2516\end{array}$ & $\begin{array}{l}1,0781 \\
+\quad 1,1640 \\
+\quad 0,1354\end{array}$ \\
\hline
\end{tabular}

Nach diesen zwei Untersuchungsergebnissen zu urteilen, liegt also. während des Höhestadiums der Krankheit noch vor der Behandlung. eine große Retention von Salzen vor, insbesonders Kalk, aber auch $\mathrm{NaCl}$ (Lust und Klocman). In der zweiten Untersuchungsperiode, während des Reparationsstadiums, ist im Gegenteil eine negative oder sehr geringe positive Salzbilanz vorhanden. Es scheint, schreiben Lust und Klocman, als wollte sich der Körper eines Depots überflüssigen Knochenmaterials entledigen, zu dessen Eliminierung er während des Höhestadiums der Erkrankung nicht die Fähigkeit hatte. Auf diese Weise können sie sich das Verhalten des Kalkes und Phosphors. erklären, die ja beide Bestandteile der zerstörten Knochen sind. Die negative Chlorbilanz findet aber in einer Störung im Bereiche der Knochen keine genügende Erklärung. Die Verfasser denken an die Möglichkeit, daß der Kalk zum Teil auch als das leicht lösliche Kalziumchlorid ausgeschieden wird. Dieser eigenartige Chlorstoffwechsel findet aber 
seine natürliche Erklärung in der hier beschriebenen Störung des Wasserhaushaltes: Wasserretention während des Höhestadiums der Erkrankung, Wasserverlust während des Reparationsstadiums. Das Wasser kann jedoch. nicht als Aqua destillata im Körper aufgespeichert werden, sondern muß immer an eine gewisse Menge von Kochsalz gebunden sein. Daher die NaCl-Retention während des Höhestadiums mit ihrer Neigung zur Wasseraufspeicherung, Gleichzeitig mit der Besserung der Krankheit tritt auch eine Hebung in der Fähigkeit des Organismus, überflüssiges Wasser auszuscheiden, ein. Es kommt zu einer Wasserflut und daher auch zu einer vermehrten Ausscheidung von Kochsalz. Damit stimmen, scheint mir, auch die Untersuchungsergebnisse von Bardt und Edelstein überein, die sie in ihren Organanalysen eines an Skorbut gestorbenen Kindes gefunden haben, nämlich $\mathrm{da} B$ die Na- und K-Menge etwas vermehrt waren, eine Tatsache, die auch diese Verfasser auf den vermehrten Wassergehalt der Organe zurückführen. $\mathrm{Ob}$ die NaCl-Retention primär und die Wasserretention sekundär oder umgekehrt die $\mathrm{NaCl}$-Retention von der Wasseransammlung bedingt sei, ist jedoch nicht durch diese Befunde entschieden. Die Kochsalzretention muß also nicht erklären, warum wir beim Säuglingsskorbut eine Wasserretention bekommen. Kochsalz- und Wasserstoffwechsel laufen nur parallel.

Während des Krieges hat man einige Erfahrungen gemacht, von denen man vielleicht hoffen konnte, daß sie auch Licht in diese Störung des Wasserhaushaltes beim Säuglingsskorbut bringen würden. Wegen der schlechten Ernährungsverhältnisse in der Kriegszeit wurden hier und da, nicht am wenigsten hier in Wien, eine große Anzahl von Skorbutfällen beobachtet. Zu gleicher Zeit trat auch eine andere Krankheit, von der man in der Friedenszeit verschont war, auf, die auch mit der Ernährung zusammenhängt: die ${ }^{*}$ Odemkrankheit.

Die Ursache dieser Ödeme schiebt man mit gutem Grunde auf eine Capillarschädigung (Schittenhelm und Schlecht, Bürger, Masse und Zondek), wodurch die Capillarwände die Blutflüssigkeit nicht mehr zurückhalten können. Man ist daher der Ansicht, daß eine noch unbekannte Noxe der Capillarwand schadet. Es kommt dabei zu einer pathologischen Durchlässigkeit derselben mit Flüssigkeitsaustritt in das Gewebe und zu einer sekundären Kochsalzretention. Eine Capillarschädigung liegt indessen auch beim Skorbut vor (Hess und Fish, Noeggerath), und der Wasserreichtum der Gewebe beim Säuglingsskorbut wird auch auf diese Funktionsstörung zurückgeführt (Morse und Talbot). Auch die hämorrhagische Diathese bei der Krankheit beruht nach Hess auf derselben Schwäche der Capillarwand, die bewirkt, daß Blutungen bei der geringsten Veranlassung zustande kommen. Unaufgeklärt bleibt jedoch, warum die geschädigte Capillarwand das 
eine Mal nur Plasmabestandteile durchläßt, ein anderes Mal das Blut als solches. Hier liegt jedenfalls eine Ähnlichkeit in der Pathogenese der Symptome bei der Ödemkrankheit und beim Skorbut vor.

Eine andere Ähnlichkeit betrifft das Herz. Bei beiden Erkran. kungen kann man eine Herzdilatation beobachten.

Die Ödemkrankheit im eigentlichen Sinne ist selten mit skorbu. tischen Symptomen kompliziert, dagegen ist es keine Seltenheit, da $B$ die Skorbutkranken eine mehr oder weniger ausgesprochene Wasseranschwellung zeigen. Asch off und Koch fanden in mehr als $2 / 3$ ihrer Fälle eine Störung des Wasser- und Salzstoffwechsels auch in den Fällen, wo gar keine Veranlassung war, kliniseh von einer Ödemkrankheit zu sprechen. Sie stützen ihre Auffassung durch Erscheinungen von allgemeiner Wassersucht. Das Bild kann sich jedoch sehr dem der Ödemkrankheit ähneln. Infolge dieser Wasserretention beim Skorbut und ihres Vorhandenseins in einem Stadium, wo die hämorrhagische Diathese wenig ausgesprochen ist, kann es auch bisweilen nach Bürger sehr.schwierig sein, diesen Zustand von einer beginnenden Ödemkrankheit zu unterscheiden. In einigen Fällen, vor allem in gewissen Gegenden konnte man indessen von einer echten Kombination zwischen Skorbut und Ödemkrankheit sprechen (Strauss, Rumpel, Falta, Wheeler, Salle und Rosenberg). Man hatte beobachtet, daß in gewissen Gefangenenlagern nur ein Teil der skorbutkranken Gefangenen Ödemkrankheit bekamen (Salle und Rosenberg). Diese verschiedenen Verhältnisse glaubte man darauf zurückführen zu können, daß die beiden Gruppen von Gefangenen aus verschiedenen Gegenden ge. kommen und verschieden ernährt worden waren. Bald gesellte sich die Ödemkrankheit zu einem schon bestehenden Skorbut, in anderen Fällen wieder erschien zuerst die Ödemkrankheit und komplizierte sich erst später mit skorbutischen Symptomen.

Daß diese beiden Erkrankungen etwas miteinander zu tun haben oder vielleicht verwandt sind, ist unwahrscheinlich. Man weiß ja, daß der Skorbut eine sogenannte Avitaminose ist. Ob aber auch die Ödemkrankheit dazu zu rechnen ist, ist jedenfalls noch unbekannt. Trotz vieler ätiologischer Forschungen über die Ödemkrankheit ist man über die Vermutung noch nicht hinausgekommen, daß sie in einer gewissen Beziehung zu einer ungeeigneten Nahrung steht, besonders zu einer einseitigen Kohlenhydratkost mit allzu wenig Eiweiß und Fett (Schittenhelm und Schlecht u. a.), eine Krankheitsursache also, die ähnlich ist der beim Mehlnährschaden der Säuglinge. Das Auftreten von einem Krankheitsbild, das in einer Kombination zwischen Ödemkrankheit und Skorbut besteht, ist nun, nach Salle und Rosenberg; auf eine kalorisch ungenügende, kohlenhydratreiche, aber vita: minarme Nahrung zurüickzuführen. In den Fällen, wo Salle und 
Rosenberg Ödeme bei den Skorbutkranken nachweisen konnten, lag auch immer eine Unterernährung vor. In diesem Sinne habe ich die Krankengeschichten meines Materials geprüft, aber ich habe mich in keinem Falle überzeugen können, daß eine deutliche Unterernährung bei den skorbutkranken Säuglingen vorgelegen ist. Nur den Kohlenhydratreichtum der Kost findet man bei beiden Erkrankungen.

Zufolge der hier erwähnten Analogien könnte man vielleicht doch geneigt sein, die Wasserretention beim Säuglingsskorbut auf dieselben Faktoren, die die Ödemkrankheit hervorrufen, zurückzuführen. Meiner Ansicht nach ist es indessen unwahrseheinlich, daß dies der Fall ist. Wenn die Wasserretention als eine larvierte Ödemkrankheit aufzufassen wäre, dann hätte man sehr große Ödeme gerade bei diesen Kindern zu erwarten, ausgesprochener noch als beim Skorbut der Erwachsenen wegen der bei Säuglingen bestehenden größeren Tendenz zu Störungen im Wasserhaushalte überhaupt. Aber nun ist gerade das Gegenteil der Fall. Im allgemeinen kommen deutliche Ödeme viel öfter beim Skorbut der Erwachsenen vor als bei Säuglingen. Auch die Eigenart der Wasserretention beim Säuglingsskorbut scheint mir gegen ihre Auffassung als zur gewöhnlichen Ödemkrankheit oder zum Mehlnährschaden gehörig zu sprechen.

Aller dieser Tatsachen wegen wäre ich am ehesten geneigt; die Störung des Wasserhaushaltes beim Säuglingsskorbut, die von mehreren Autoren beschrieben ist, als ein Teilsymptom der Skorbuterkrankung als solcher zu betrachten. Der Zuistand des Organismus, der entsteht, wenn das antiskorbutische Vitamin fehlt, bewirkt wahrscheinlich neben den bereits wohlbekannten Skelettveränderungen und der hämorrhagischen Diatheso auch eine vermehrte Durchlässigkeit der Capillarwände und eine Umstimmung der Körperzellen, um diese ausgetretenen Wassermengen zu assimilieren. Ich habe oben die oft relativ hohe Kohlenhydraternährung der Barlowkinder hervorgehoben. Diese und vielleicht auch andere ungeeignete Ernährungen sind im allgemeinen doch nicht so weit vom Normalen entfernt, daß sie an und für sich eine erhöhte Wasserretention hervorbringen könnten, aber sie bewirken vielleicht, daß die Wasserretention leichter zustande kommt.

\section{Literaturverzeichnis.}

A sch off und Koch, Zur pathologischen Anatomie und Histologie des Skorbuts. Dtsch. med. Wochenschr. 1918, S. 423. - Bardt und Edels tein, Organanalysen bei Barlowscher Krankheit. Zeitschr. f. Kinderheilk., 9, 414. 1913. - Barlow Tho mas, Skorbut infantile in Traité des Malad. de l'enfance von Grancher, Comby und Marfan. 1897, Teil II. S. 173. - Bürger, Max, Die Ödemkrankheit. Ergebn. d. inn. Med. u. Kinderh., 18, 1920. - Czerny, Adolf, Über die Bedeutung des Turgordruckes der Gewebe für das Kind im ersten Lebensjahre. Monatsschr. f. Kinderheilk., I, I, l. 1902. - Epstein, Aloi s, Über eine auffällige Häufung der 
Barlowschen Krankheit in den Kriegsjahren 1917-1918. Jahrb. f. Kinderheilk. 88, 1918. - Falta, W., Über das Kriegsödem. Wien. klin. Wochenschr. 1917, Nr. 52, S. 1637. - Fran k, M., Beitrag zur Klinik und zum Stoffwechsel der MöllerBarlowschen Krankheit. Jahrb. f. Kinderheilk., 91, 21.1920. -Fre und, Walther, Wasser und Salze in ihren Beziehungen zu den Körpergewichtsschwankungen der Säuglinge. Jahrb. f. Kinderheilk., 59, 420. 1904. - Fürst, L., Die Barlowsche Krankheit. Arch. f. Kinderhcilk., 18, 50. 1895. - Hess, Alfred und Fish, Mildred, Infantil Seurvy: The Blood, The Bloodplatelets and the Diet. The am. Journ. of dis. of Childr. 8, 386. 1914. - Hess, Alfred, A new aspect of the symp. tomatology, pathology and diet (infantil scurvy). The journ. of the am. med. ass. 65, 1003. 1915. - Hess, Alfred, Infantil Scurvy. III. Its Influence on Growth (Length and Weight). The am. journ. of dis. of Childr. 12, 152. 1916. - Hess, Alfred, Subacute and Latent Infantil Scurvy. The journ. of the am. med. ass., 63, 235. 1917. - Hess, Alfred, Infantil Seurvy. V. A Study of its Pathogenesis. The am. journ. of Dis. of Childr. 14, 337. 1917. - Lust, F. und L. Klocman, Stoffwechselversuche bei Barlowscher Krankheit. Jahrb. f. Kinderheilk., 75, 663. 1920. - Maase, C. und H. Zondek, Das Hungerödom. Leipzig 1920. - Me yer, L. F., Über den Wasserbedarf des Säuglings. Zeitschr. f. Kinderheilk., 5, 1. 1913. - Meyer, L. F., Idiopathische Ödeme im Kindesalter. Ergebn. d. inn. Med. u. Kinderheilk., 17, 562. 1919. - Morse, John Lovell und Fritz Talbot, Diseases of nutrition and infant fecding. II. edit. New Yolk 1920. - Nobel, Fdmund, Beitrag zur Barlowfrage. Wien. klin. Wochenschr. 1920, Nr. 52. Nobel, Edmund, Zur Barlowfrage. Bedeutung der Hitzewirkung auf die Vitamine. Beitrag zur Frage der Nahrungskonzentration. Zeitschr. f. Kinderheilk., 28, 3. 1921. - Nobel, Ed m und und Richard Wagner, Trockenmilch in der Kinderernährung. Zeitsehr. f. Kinderheilk. 1921. - Ru m pel, Th., Zur Ätiologie der Ödemkrankheiten in russischen Gefangenenlagern. Münohn. med. Wochenschr. (Feldärzt. Beilage) 1915, Nr. 30, S. 1021. - Salle, Vikt or und Max Rosenberg, Über Skorbut. Ergebn. d. inn. Med. u. Kinderheilk., 19. 1921. - Schittenhel m, A. und H. Schlecht, Über die Ödemkrankheit. Zeitschr. f. d. ges. exper. Med. 9, 1. 1919. - Stoeltz ner, W., Ein anatomisch untersuchter Fall von Barlowscher Krankheit. Jahrb. f. Kinderheilk., 63, 36. 1906. - Stra uss, Die Hungerkrankheit. Med. Klinik 1915, Nr. 31, S. 854.

Wien, Universitäts-Kinderklinik. 